\title{
Editorial
}

\section{Detection and treatment of early gastric cancer in the West}

\author{
H.M. SUE-LING \\ Division of Surgery and Centre for Digestive Diseases, The General Infirmary, Leeds LS1 3EX, United Kingdom \\ e-mail 1.o.harrison@leeds.ac.uk
}

\section{Introduction}

Although gastric cancer is declining in incidence in the West, it is still the second commonest cause of death from cancer in the world and the fourth commonest cause in Britain. In the United Kingdom alone, approximately 100000 people died of gastric cancer in the 1980 s, and the 5 -year survival was a dismal 5\%-6\%. The poor prognosis of the disease in Britain and much of the West is largely due to the fact that gastric cancer is often diagnosed too late. In the largest populationbased survey [1] of gastric cancer ever undertaken in Britain, $80 \%$ of patients were found to have advanced and incurable stage IV disease, and only $1 \%$ had early gastric cancer. The crucial factor, then, is earlier diagnosis if the results of treatment of gastric cancer are to improve. Advances in surgical techniques are of little account if the presence of gastric cancer is detected too late.

\section{Detection of early gastric cancer}

Screening for gastric cancer is an attempt to pick up the disease either at a pre-malignant stage or at a sufficiently early stage which would allow curative treatment. In Japan, mass screening of asymptomatic people by radiological means has proved effective in diagnosing gastric cancer at an early pathological stage. Where such mass screening programmes have been implemented, the proportion of patients diagnosed with early gastric cancer has risen steadily, from approximately $20 \%$ in the 1970 s to $50 \%-60 \%$ in the 1990 s [2]. In the West, however, the lower incidence of gastric cancer renders such a screening programme impractical.

Offprint requests to: H.M. Sue-Ling Received for publication on Jul. 27, 1998; accepted on Aug. 1, 1998
Nevertheless, it is still possible to identify high-risk groups of patients who should be referred early for investigation by means of endoscopy and biopsy. The first group of high-risk patients are those with conditions such as pernicious anaemia, gastric ulcer, chronic atrophic gastritis, intestinal metaplasia, or those who have had partial gastrectomy or vagotomy and gastroenterostomy in the past. The second and perhaps more important group of patients are those with dyspepsia who are over 40 years of age. It is estimated that approximately $30 \%$ of the population suffer from dyspepsia at any time and, of these, a third will consult their general practitioner. Patients over the age of 40-45 years with dyspepsia have a 1 in 50 chance of having gastric cancer. ${ }^{3}$ If such patients are referred promptly, a quarter of the gastric cancers detected will be early gastric cancers and over half of the patients would be suitable for potentially curative resection $[3,4]$.

In the past, great disservice to the cause of early diagnosis of gastric cancer has been rendered by teachers at medical schools who emphasise the clinical features of advanced and incurable disease. Every medical student seems to know about cachexia, Virchow's node, epigastric mass, hepatomegaly, ascites and such features of advanced and usually incurable stage-IV disease which have even been used in computer-assisted programmes for the diagnosis of gastric cancer. It is important that medical students should be taught that the symptoms of early, curable gastric cancer are virtually indistinguishable from the symptoms of benign peptic ulcer disease, gastritis or reflux oesophagitis. With regard to the signs of gastric cancer, ideally there should be none at all. Thus, every patient over 40 years of age who consults their general practitioner complaining of dyspepsia or pain, nausea, vomiting, dysphagia, with or without weight loss, should be investigated promptly by means of endoscopy, with biopsy of any suspicious lesion. Likewise, all patients with apparently benign gastric ulcers, iron deficiency anaemia or who belong to 
other high-risk groups, should be investigated similarly. Procrastination, in the form of a so-called therapeutic trial of anti-ulcer medication, may alleviate the patient's symptoms for several months and delay the diagnosis of gastric cancer, so that the golden opportunity is lost.

A positive and vigorous approach to the diagnosis of gastric cancer such as that outlined above has been described in several centres in Europe, with encouraging results [3-6]. Diagnosis of gastric cancer at an early stage must remain our number one priority if the outlook for patients with gastric cancer is to improve. The essential factor is that general practitioners and hospital doctors alike should be alert to the possible presence of gastric cancer in any patient over the age of 40 years who complains of dyspepsia or is anaemic, and prompt investigation by means of endoscopy and biopsy should be instituted.

\section{Treatment of early gastric cancer}

It is debatable whether or not all patients with early gastric cancer should undergo radical surgical resection with extended, Japanese-style D2 lymph node dissection. It could be argued that any slight gain in terms of survival achieved by radical surgery may be offset by an increase in operative mortality and morbidity. The incidence of lymphatic metastases is directly related to the depth of invasion of the primary tumour, ranging from $4 \%$ for tumours confined to the mucosa to $20 \%$ for tumours extending to the submucosa [7]. Thus, it would seem logical to carry out a more conservative (D1) resection with complete excision of perigastric N1 nodes in patients with early gastric cancer confined to the mucosa, reserving the more radical (D2) resection for patients in whom the tumour has penetrated to the sub-mucosa.

In Japan, with the help of very sophisticated imaging techniques, including the use of dye sprays, endoluminal ultrasound scan and even arteriograms, it is possible to accurately distinguish between mucosal and submucosal tumours before operation. This has led to a more conservative line of treatment of early gastric cancers in Japan, with endomucosal resection or limited surgery for small mucosal tumours less than $2 \mathrm{~cm}$ in size. Larger tumours or those that have penetrated to the submucosa are still treated by the classical D2 resection.

In the West, however, such a strategy may still be difficult to implement. Assessment of the depth of invasion of early gastric cancer would require the use of endoluminal ultrasound scan, which is still not widely available in many centres in the West. Even if it were available, accurate distinction between mucosal and submucosal tumours may still not be possible, as most early gastric cancers seen in the West are ulcerated tumours [4], with the presence of fibrosis in the submucosa and underlying muscularis propria making it difficult to distinguish between mucosal and submucosal tumours. The small type 1 mucosal tumours that are commonly seen in Japan as a result of mass screening are seldom seen in the West. It is for these reasons that most patients who appear at operation to have an "early" gastric cancer should still undergo a radical resection with extended D2 lymph node dissection, as Professor Oliveira and colleagues emphasize in this issue [8], with perhaps preservation of the pancreas and spleen whenever possible to reduce operative mortality and post-operative morbidity [9].

\section{Summary}

In conclusion, the traditional Western view that gastric cancer is usually a fatal disease is out of date. The poor prognosis of gastric cancer in the West should no longer be accepted as inevitable. To detect gastric cancer at an early stage, what is required is the education of doctors and the public alike that the onset of symptoms of pain, dyspepsia or anaemia in patients over 40 years of age should be investigated promptly by means of endoscopy and biopsy. Surgical treatment of patients with early gastric cancer should ideally be concentrated in specialised units and should take the form of a radical $\mathrm{D} 2$ resection with preservation of spleen and pancreas whenever possible.

\section{References}

1. Allum WH, Powell DJ, McConkey CC, Fielding JWL. Gastric cancer: A 25 year review. Br J Surg 1989;76:535-40.

2. Maruyama K, Okabayashi K, Kinoshita T. Progress in gastric cancer surgery in Japan and its limits of radicality. World J Surg 1987;11:418-25.

3. Hallissey MT, Allum WH, Jewkes AJ, Ellis DJ, Fielding JWL. Early detection of gastric cancer. BMJ 1990;301:513-5.

4. Sue-Ling HM, Martin I, Griffith J, Ward DC, Quirke P, Johnston $\mathrm{D}$, et al. Early gastric cancer: 46 patients treated in one surgical department. Gut 1992;33:1318-22.

5. Gear MWL, Ormiston MC, Barnes RJ, Rocyn-Jones J, Voss GC. Endoscopic studies of dyspepsia in the community: An "openaccess" service. BMJ 1980;280:1135.

6. Oleagoitia JM, Eschevarria A, Santidrian JI, Ulacla MA, Hernandez-Calvo J. Early gastric cancer. Br J Surg 1986;73:804-6.

7. Fukutomi H, Sakita T. Analysis of early gastric cancer cases collected from major hospitals and institutes in Japan. Jpn J Clin Oncol 1984;14:169-79.

8. Oliveira FJ, Ferrão H, Furtado E, Batista H, Conceição L. Early gastric cancer: Report of 58 cases. Gastric Cancer 1998;1:516.

9. Griffith J, Sue-Ling HM, Johnston D, et al. Preservation of spleen improves survival after radical surgery for gastric cancer. Gut 1995;36:684-90. 